\title{
ON CENTRAL COMMUTATOR GALOIS EXTENSIONS OF RINGS
}

\author{
GEORGE SZETO and LIANYONG XUE
}

(Received 17 November 1999)

\begin{abstract}
Let $B$ be a ring with $1, G$ a finite automorphism group of $B$ of order $n$ for some integer $n, B^{G}$ the set of elements in $B$ fixed under each element in $G$, and $\Delta=V_{B}\left(B^{G}\right)$ the commutator subring of $B^{G}$ in $B$. Then the type of central commutator Galois extensions is studied. This type includes the types of Azumaya Galois extensions and Galois $H$-separable extensions. Several characterizations of a central commutator Galois extension are given. Moreover, it is shown that when $G$ is inner, $B$ is a central commutator Galois extension of $B^{G}$ if and only if $B$ is an $H$-separable projective group ring $B^{G} G_{f}$. This generalizes the structure theorem for central Galois algebras with an inner Galois group proved by DeMeyer.
\end{abstract}

Keywords and phrases. Azumaya algebras, Galois extensions, central Galois extensions, Azumaya Galois extensions, center Galois extensions, $H$-separable extensions.

2000 Mathematics Subject Classification. Primary 16S35, 16W20.

1. Introduction. Galois theory for commutative rings were studied in the sixties and seventies (see [4, Chapter 3]), and several Galois extensions of noncommutative rings were also investigated (see [2, 5, 6, 8]). Recently, central Galois extensions and the DeMeyer-Kanzaki Galois extensions were generalized to the Azumaya Galois extensions and center Galois extensions, respectively (see $[1,9,10,11]) . B$ is called an Azumaya Galois extension of $B^{G}$ with Galois group $G$ if $B$ is a Galois extension of $B^{G}$ which is an Azumaya algebra over $C^{G}$ where $C$ is the center of $B$, and $B$ is called a center Galois extension of $B^{G}$ if $C$ is a Galois algebra with Galois group $\left.G\right|_{C} \cong G$. The purpose of the present paper is to study a type of Galois extensions which is strictly between the types of Azumaya Galois extensions and Galois $H$-separable extensions. Let $\Delta=V_{B}\left(B^{G}\right)$, the commutator subring of $B^{G}$ in $B$. We call $B$ a commutator Galois extension of $B^{G}$ if $\Delta$ is a Galois extension with Galois group $\left.G\right|_{\Delta} \cong G$, and $B$ is a central commutator Galois extension of $B^{G}$ if $\Delta$ is a central Galois algebra with Galois group $\left.G\right|_{\Delta} \cong G$. We shall characterize a central commutator Galois extension in terms of a Galois $H$-separable extension $B$ of $B^{G}$ as studied by Sugano (see [8]) and the $C$-modules $\left\{J_{g} \mid g \in G\right\}$ where $J_{g}=\{b \in B \mid b a=g(a) b$ for all $a \in B\}$. Moreover, it will be shown that $B$ is a central commutator Galois extension of $B^{G}$ with an inner Galois group $G$ if and only if $B$ is an $H$-separable projective group ring $B^{G} G_{f}$ where $B^{G} G_{f}=\sum_{g \in G} B^{G} U_{g}$ such that $\left\{U_{g} \mid g \in G\right\}$ are free over $B^{G}, b U_{g}=U_{g} b$ for all $b \in B^{G}$ and $g \in G$, and $U_{g} U_{h}=U_{g h} f(g, h)$ where $f: G \times G \rightarrow$ units of $C^{G}$ is a factor set. This generalizes the structure theorem for a central Galois algebra with an inner Galois group proved by DeMeyer (see [3]). 
2. Basic definitions and notation. Throughout this paper, $B$ will represent a ring with $1, C$ the center of $B, G$ a finite automorphism group of $B$ of order $n$ for some integer $n, B^{G}$ the set of elements in $B$ fixed under each element in $G$, and $\Delta=V_{B}\left(B^{G}\right)$, the commutator subring of $B^{G}$ in $B$.

Let $A$ be a subring of a $\operatorname{ring} B$ with the same identity 1 . We call $B$ a separable extension of $A$ if there exist $\left\{a_{i}, b_{i}\right.$ in $B, i=1,2, \ldots, m$ for some integer $\left.m\right\}$ such that $\sum a_{i} b_{i}=1$, and $\sum b a_{i} \otimes b_{i}=\sum a_{i} \otimes b_{i} b$ for all $b$ in $B$ where $\otimes$ is over $A$, and a ring $B$ is called an $H$-separable extension of $A$ if $B \otimes_{A} B$ is isomorphic to a direct summand of a finite direct sum of $B$ as a $B$-bimodule. An Azumaya algebra is a separable extension of its center. $B$ is called a Galois extension of $B^{G}$ with Galois group $G$ if there exist elements $\left\{c_{i}, d_{i}\right.$ in $\left.B, i=1,2, \ldots, m\right\}$ for some integer $m$ such that $\sum_{i=1}^{m} c_{i} g\left(d_{i}\right)=\delta_{1, g}$ for $g \in G$. The set $\left\{c_{i}, d_{i}\right\}$ is called a $G$-Galois system for $B$. $B$ is called a DeMeyerKanzaki Galois extension of $B^{G}$ if $B$ is an Azumaya $C$-algebra and $C$ is a Galois algebra with Galois group $\left.G\right|_{C} \cong G$. If $C$ is a Galois algebra with Galois group $\left.G\right|_{C} \cong G$, we call $B$ a center Galois extension of $B^{G} . B$ is called an Azumaya Galois extension if it is a Galois extension of $B^{G}$ that is an Azumaya $C^{G}$-algebra, and $B$ is called a Galois $H$-separable extension if it is a Galois and an $H$-separable extension of $B^{G}$ (see [8]). We call $B$ a commutator Galois extension of $B^{G}$ if $\Delta$ is a Galois extension with Galois group $\left.G\right|_{\Delta} \cong$ $G$, and $B$ is a central commutator Galois extension of $B^{G}$ if $\Delta$ is a central Galois algebra with Galois group $\left.G\right|_{\Delta} \cong G$. For each $g \in G$, let $J_{g}=\{b \in B \mid b x=g(x) b$ for all $x \in B\}$ and $J_{g}^{A}=\{a \in A \mid a x=g(x) a$ for all $x \in A\}$ for a subring $A$ of $B$.

3. Central commutator Galois extensions. In this section, we shall give several characterizations of a central commutator Galois extension in terms of Galois $\mathrm{H}$ separable extensions and Azumaya Galois extensions, respectively, and prove the converse of a theorem for a Galois $H$-separable extension as given in [8]. We begin with some properties of a commutator Galois extension.

LEMMA 3.1. If $B$ is a commutator Galois extension of $B^{G}$, then $\Delta$ is a Galois algebra over $C^{G}$.

Proof. Since $\Delta$ is a Galois extension of $\Delta^{G}$ with Galois group $\left.G\right|_{\Delta} \cong G, B$ and $B^{G} \Delta$ are also Galois extensions of $B^{G}$ with Galois group $G$ and $\left.G\right|_{B^{G} \Delta}$. But $B^{G} \Delta \subset B$ and $\left.G \cong G\right|_{B^{G} \Delta}$, so $B=B^{G} \Delta$. Thus, the center of $\Delta$ is $C$; and so $\Delta^{G}=B^{G} \cap \Delta=C^{G}$.

LEMMA 3.2. If B is a commutator Galois extension of $B^{G}$, then $J_{g}=J_{g}^{\Delta}$ for each $g \in G$.

Proof. Since $J_{g}=\{b \in B \mid b a=g(a) b$ for all $a \in B\} \subset\{b \in B \mid b a=g(a) b$ for all $\left.a \in B^{G}\right\}=\Delta, J_{g} \subset J_{g}^{\Delta}$.

Conversely, for any $x \in J_{g}^{\Delta}, x d=g(d) x$ for all $d \in \Delta$. Since $\Delta$ is a Galois extension of $\Delta^{G}$ with Galois group $\left.G\right|_{\Delta} \cong G, B=B^{G} \Delta$ by the proof of Lemma 3.1. So for any $b \in B, b=\sum_{i=1}^{m} b_{i} d_{i}$ for some $b_{i} \in B^{G}, d_{i} \in \Delta$ and some integer $m$, we have that $x b=$ $x \sum_{i=1}^{m} b_{i} d_{i}=\sum_{i=1}^{m} b_{i} x d_{i}=\sum_{i=1}^{m} b_{i} g\left(d_{i}\right) x=g\left(\sum_{i=1}^{m} b_{i} d_{i}\right) x=g(b) x$. Thus, $J_{g}^{\Delta} \subset J_{g}$; and so $J_{g}=J_{g}^{\Delta}$.

THEOREM 3.3. The following are equivalent:

(1) $B$ is a central commutator Galois extension of $B^{G}$. 
(2) $B$ is a commutator Galois extension of $B^{G}$ and $J_{g} J_{g^{-1}}=C$ for each $g \in G$.

(3) $B$ is a Galois $H$-separable extension of $B^{G}, B=B^{G} \Delta$, and $n^{-1} \in B$.

Proof. $(1) \Rightarrow(2)$. It is clear.

(2) $\Rightarrow(1)$. By Lemma 3.1, $\Delta^{G}=C^{G}$, so $\Delta$ is a Galois algebra with Galois group $\left.G\right|_{\Delta} \cong G$. By hypothesis, $J_{g} J_{g^{-1}}=C$ for each $g \in G$ and by Lemma 3.2, $J_{g}=J_{\mathscr{g}}^{\Delta}$ for each $g \in G$, so $\Delta$ is a central Galois algebra (see [5, Theorem 1]).

$(1) \Rightarrow(3)$. Since $\Delta$ is a central Galois $C^{G}$-algebra, we have $B=B^{G} \Delta, J_{g}=J_{g}^{\Delta}$ for each $g \in G$ by Lemma 3.2 and $J_{g}^{\Delta} J_{g^{-1}}^{\Delta}=C$ (see [6, Lemma 2]). Hence $J_{g} J_{g^{-1}}=C$ for each $g \in G$. But $B$ is a Galois extension of $B^{G}$ with the same Galois system for $\Delta$, so $B$ is a Galois $H$-separable extension of $B^{G}$ (see [8, Theorem 2(iii) $\Rightarrow$ (i)]). Moreover, $n^{-1} \in B$ (see [6, Corollary 3]), so (3) holds.

(3) $\Rightarrow(1)$. Since $B=B^{G} \Delta$, the group $H=\left\{g \in G|g|_{\Delta}\right.$ is an identity $\}=\{1\}$. Thus, $\Delta$ is a central Galois algebra over $\Delta^{G}$ (see $\left[8\right.$, Theorem 6 , (3)(ii) $\Rightarrow\left(\right.$ iii)]) where $\Delta^{G}=C^{G}$ by Lemma 3.1.

We remark that $(1) \Rightarrow(3)$ in Theorem 3.3 is the converse of [8, Theorem 6]; that is, if $\Delta$ is a central Galois algebra with Galois group $\left.G\right|_{\Delta} \cong G$, then

(i) $n^{-1} \in B$,

(ii) $B=B^{G} \Delta$,

(iii) $B$ is a Galois $H$-separable extension of $B^{G}$.

In the next theorem, we give a characterization of a central commutator Galois extension in terms of Azumaya Galois extensions.

THEOREM 3.4. The following are equivalent:

(1) $B$ is a central commutator Galois extension of $B^{G}$ and $B^{G}$ is a separable $C^{G}$-algebra.

(2) $B$ is an Azumaya Galois extension with Galois group $G$.

(3) $B$ is a central commutator Galois extension and a separable extension of $\Delta$.

Proof. $\quad(1) \Rightarrow(2)$. Since $B$ is a central commutator Galois extension, $B$ is a Galois $H$ separable extension of $B^{G}$ by Theorem 3.3(3). Thus, $V_{B}\left(V_{B}\left(B^{G}\right)\right)=B^{G}$ (see [8, Proposition 4(1)]). This implies that $C \subset B^{G}$; and so $C=C^{G}$. Moreover, by Theorem 3.3(3) again, $B=B^{G} \Delta$, so the center of $B^{G}$ is $C^{G}$, the center of $B$. Thus, $B^{G}$ is an Azumaya $C^{G}$-algebra. By noting that $B$ is a Galois extension of $B^{G},(2)$ holds.

$(2) \Rightarrow(1)$. It is a consequence of [1, Lemma 1].

$(1) \Rightarrow(3)$. Since $B$ is a separable extension of $B^{G}$ (for it is a Galois extension) and $B^{G}$ is a separable $C^{G}$-algebra, $B$ is a separable $C^{G}$-algebra by the transitivity property of separable extensions. Thus, $B$ is a separable extension of $\Delta$ because $C^{G} \subset \Delta \subset B$.

(3) $\Rightarrow(1)$. Since $\Delta$ is a Galois extension of $\Delta^{G}$ with Galois group $\left.G\right|_{\Delta} \cong G, \Delta$ is a separable extension of $\Delta^{G}$. By Lemma 3.1, $\Delta^{G}=C^{G}=C$ (for $C$ is the center of $\Delta$ ). By hypothesis, $B$ is a separable extension of $\Delta$. Hence $B$ is a separable extension of $C$, that is, $B$ is an Azumaya $C$-algebra. By Lemma 3.1 again, $B=B^{G} \Delta$ such that $B^{G}$ and $\Delta$ are $C$-subalgebras of the Azumaya $C$-algebra $B$. Hence, they are Azumaya $C$-algebras by the commutator theorem for Azumaya algebras (see [4, Theorem 4.3, page 57]). Since $\Delta$ is a Galois extension of $\Delta^{G}$ with Galois group $\left.G\right|_{\Delta} \cong G, B$ is a Galois extension of $B^{G}$ which is an Azumaya $C^{G}$-algebra. This completes the proof. 
4. $H$-separable projective group rings. In [3], it was shown that $B$ is a central Galois algebra with an inner Galois group $G$ if and only if $B$ is an Azumaya projective group algebra $C^{G} G_{f}$ over $C^{G}$ where $C^{G} G_{f}=\sum_{g \in G} C^{G} U_{g}$ such that $\left\{U_{g} \mid g \in G\right\}$ are free over $C^{G}, c U_{g}=U_{g} c$ for all $c \in C^{G}$ and $g \in G$, and $U_{g} U_{h}=U_{g h} f(g, h), f: G \times G \rightarrow$ units of $C^{G}$ is a factor set (see [3]). We shall generalize this fact to a central commutator Galois extension with an inner Galois group.

THEOREM 4.1. $B$ is a central commutator Galois extension of $B^{G}$ with an inner Galois group $G$ if and only if $B=B^{G} G_{f}$ which is an $H$-separable extension of $B^{G}$ and $n^{-1} \in B$.

Proof. $\quad \Leftrightarrow)$ By Theorem $3.3(1) \Rightarrow(3), B=B^{G} \Delta$ which is a Galois $H$-separable extension of $B^{G}$ and $n^{-1} \in B$, so it suffices to show that $B=B^{G} G_{f}$, a projective group ring with coefficient ring $B^{G}$. Since $\Delta$ is a central Galois $C^{G}$-algebra, by [3, Theorem 2], $\Delta=C^{G} G_{f}$, a projective group algebra over $C^{G}$ where $f: G \times G \rightarrow$ units of $C^{G}$ is a factor set such that $f(g, h)=U_{g} U_{h} U_{g h}^{-1}$ for all $g, h \in G$. Noting that $b U_{g}=U_{g} b$ for all $b \in B^{G}$ and $g \in G$, we claim that $\left\{U_{g} \mid g \in G\right\}$ are independent over $B^{G}$. Assume $\sum_{g \in G} b_{g} U_{g}=0$ for some $b_{g} \in B^{G}$ and $g \in G$. Since $\Delta$ is a Galois extension of $\Delta^{G}$ with Galois group $\left.G\right|_{\Delta} \cong G$, there exists a $G$-Galois system $\left\{c_{i}, d_{i}, i=1,2, \ldots, m\right.$ for some integer $\left.m\right\}$ for $\Delta$ such that $\sum_{i=1}^{m} c_{i} g\left(d_{i}\right)=\delta_{1, g}$ for $g \in G$. Hence

$$
\begin{aligned}
b_{1} & =\sum_{g \in G} \delta_{1, g} b_{g} U_{g}=\sum_{g \in G} \sum_{i=1}^{m} c_{i} g\left(d_{i}\right) b_{g} U_{g} \\
& =\sum_{g \in G} \sum_{i=1}^{m} c_{i} b_{g} g\left(d_{i}\right) U_{g}=\sum_{g \in G} \sum_{i=1}^{m} c_{i} b_{g} U_{g} d_{i} \\
& =\sum_{i=1}^{m} c_{i}\left(\sum_{g \in G} b_{g} U_{g}\right) d_{i}=0 .
\end{aligned}
$$

So $\sum_{g \in G} b_{g} U_{g}=0$ for some $b_{g} \in B^{G}$ and $g \in G$ implies that $b_{1}=0$. Now for any $h \in G$, since $\sum_{g \in G} b_{g} U_{g}=0,0=\sum_{g \in G} b_{g} U_{g} U_{h^{-1}}=\sum_{g \in G} b_{g} f\left(g, h^{-1}\right) U_{g h^{-1}}$. Thus, $b_{h} f\left(h, h^{-1}\right)=0$, and so $b_{h}=0$. This proves that $\left\{U_{g} \mid g \in G\right\}$ are independent over $B^{G}$.

$(\Leftrightarrow)$ Since $B^{G} G_{f}\left(\cong B^{G} \otimes_{C^{G}} C^{G} G_{f}\right)$ is an $H$-separable extension of $B^{G}$ and $B^{G}$ is a direct summand of $B^{G} G_{f}$ as a left $B^{G}$-module, $V_{B^{G} G_{f}}\left(V_{B^{G} G_{f}}\left(B^{G}\right)\right)=B^{G}$. This implies that the center of $B^{G} G_{f}$ is $C^{G}$. Moreover, $G$ is inner induced by $\left\{U_{g} \mid g \in G\right\}$, so $J_{g}=C^{G} U_{g}$ for each $g \in G$. But then $C^{G} G_{f}=\oplus \sum_{g \in G} C^{G} U_{g}=\oplus \sum_{g \in G} J_{g}$ such that $J_{g} J_{g^{-1}}=\left(C^{G} U_{g}\right)\left(C^{G} U_{g^{-1}}\right)=C^{G}$ for all $g \in G$. By hypothesis, $n^{-1} \in C^{G}$, so $C^{G} G_{f}$ is a separable algebra over $C^{G}$. Thus, $\Delta\left(=C^{G} G_{f}\right)$ is a central Galois algebra (see [5, Theorem 1]) with an inner Galois group $\bar{G}$ induced by $\left\{U_{g} \mid g \in G\right\}$. Thus, $B$ is a central commutator Galois extension of $B^{G}$ with an inner Galois group $G$.

By [7, Theorem 1.2], we derive a one-to-one correspondence between some sets of separable subextensions in a central commutator Galois extension $B$ of $B^{G}$. Let $\mathscr{S}=$ $\left\{A \mid A\right.$ is a separable subextension of $B$ containing $B^{G}$ which is a direct summand of $B$ as a bimodule $\}$ and $\mathscr{T}=\left\{\mathscr{D} \mid \mathscr{D}\right.$ is a separable subalgebra of $\Delta$ over $\left.C^{G}\right\}$.

THEOREM 4.2. Let $B$ be a central commutator Galois extension of $B^{G}$. Then, there exists a one-to-one correspondence between $\mathscr{S}$ and $\mathcal{T}$ by $A \rightarrow V_{B}(A)$. 
Proof. By Theorem 3.3(3), $B$ is an $H$-separable extension of $B^{G}$, so the correspondence holds by [7, Theorem 1.2].

We conclude this paper with two examples of Galois extension $B$ to show that

(1) $B$ is a central commutator Galois extension but not an Azumaya Galois extension (see Theorem 3.4),

(2) $B$ is a Galois $H$-separable extension but not a central commutator Galois extension (see Theorem 3.3).

EXAMPLE 4.3. Let $A=Q[i, j, k]$ be the quaternion algebra over the rational field $Q$, $B=\left\{\left(\begin{array}{cc}a_{1} & a_{2} \\ 0 & a_{3}\end{array}\right) \mid a_{1}, a_{2}, a_{3} \in A\right\}$, the ring of all 2-by-2 upper triangular matrices over $A$ and $G=\left\{1, g_{i}, g_{j}, g_{k}\right\}$ where $g_{i}(a)=i a i^{-1}, g_{j}(a)=j a j^{-1}, g_{k}(a)=k a k^{-1}$ for all $a$ in $A$ and $g\left(\begin{array}{cc}a_{1} & a_{2} \\ 0 & a_{3}\end{array}\right)=\left(\begin{array}{cc}g\left(a_{1}\right) & g\left(a_{2}\right) \\ 0 & g\left(a_{3}\right)\end{array}\right)$ for $g \in G$. Then

(1) $A^{G}=Q$.

(2) $B^{G}=\left\{\left(\begin{array}{cc}q_{1} & q_{2} \\ 0 & q_{3}\end{array}\right) \mid q_{1}, q_{2}, q_{3} \in Q\right\}$, the ring of all 2-by-2 upper triangar matrices over $Q$.

(3) $\Delta=V_{B}\left(B^{G}\right)=\left\{\left(\begin{array}{ll}a & 0 \\ 0 & a\end{array}\right) \mid a \in A\right\} \cong A$.

(4) $\Delta$ is a Galois extension of $\Delta^{G}$ with Galois group $\left.G\right|_{\Delta} \cong G$ and a Galois system $\{1, i, j, k ; 1 / 4,-i / 4,-j / 4,-k / 4\}$.

(5) $\Delta^{G}=Q$ is the center of $\Delta$.

(6) By (4) and (5), $B$ is a central commutator Galois extension of $B^{G}$.

(7) The center of $B^{G}$ is $Q$.

(8) $B^{G}$ is not a separable extension of its center $Q$, and so $B^{G}$ is not an Azumaya algebra. In fact, suppose that $B^{G}$ is a separable extension of $Q$. Then, there exists a separable idempotent

$$
e=\sum_{\substack{1 \leq i \leq j \leq 2 \\ 1 \leq k \leq l \leq 2}} q_{i j k l}\left(e_{i j} \otimes e_{k l}\right),
$$

where $e_{11}=\left(\begin{array}{ll}1 & 0 \\ 0 & 0\end{array}\right), e_{12}=\left(\begin{array}{ll}0 & 1 \\ 0 & 0\end{array}\right), e_{22}=\left(\begin{array}{ll}0 & 0 \\ 0 & 1\end{array}\right)$, and $q_{i j k l} \in Q$ such that

$$
\sum_{\substack{1 \leq i \leq j \leq 2 \\ 1 \leq k \leq l \leq 2}} q_{i j k l} e_{i j} e_{k l}=I_{2},
$$

the identity 2-by-2 matrix, and $b e=e b$ for all $b \in B^{G}$. By $e_{11} e=e e_{11}$, we have

$$
\sum_{\substack{1 \leq j \leq 2 \\ 1 \leq k \leq l \leq 2}} q_{1 j k l}\left(e_{1 j} \otimes e_{k l}\right)=\sum_{1 \leq i \leq j \leq 2} q_{i j 11}\left(e_{i j} \otimes e_{11}\right) .
$$

Hence $q_{2211}=0$ and $q_{1 j k 2}=0$ for all $j, k$, that is, $q_{1112}=q_{1122}=q_{1212}=q_{1222}=0$. By $e_{12} e=e e_{12}$, we have

$$
\sum_{1 \leq k \leq l \leq 2} q_{22 k l}\left(e_{12} \otimes e_{k l}\right)=\sum_{1 \leq i \leq j \leq 2} q_{i j 11}\left(e_{i j} \otimes e_{12}\right) .
$$

Hence $q_{22 k l}=0$ if $(k, l) \neq(1,2)$ and $q_{i j 11}=0$ if $(i, j) \neq(1,2)$, that is, $q_{2211}=q_{2222}=0$ and $q_{1111}=q_{2211}=0$. Therefore, $e=q_{1211}\left(e_{12} \otimes e_{11}\right)+q_{2212}\left(e_{22} \otimes e_{12}\right)$. Thus,

$$
I_{2}=\sum_{\substack{1 \leq i \leq j \leq 2 \\ 1 \leq k \leq l \leq 2}} q_{i j k l} e_{i j} e_{k l}=q_{1211} e_{12} e_{11}+q_{2212} e_{22} e_{12}=0 .
$$


This contradiction shows that $B^{G}$ is not a separable extension of $Q$.

EXAMPLE 4.4. Let $B=Q[i, j, k]$ be the quaternion algebra over the rational field $Q$ and $G=\left\{1, g_{i}\right\}$ where $g_{i}(x)=i x i^{-1}$ for all $x$ in $B$. Then

(1) $B$ is a Galois extension of $B^{G}$ with Galois group $G$ and a Galois system $\{1, i, j, k ; 1 / 4$, $-i / 4,-j / 4,-k / 4\}$.

(2) Since $G$ is inner, $B$ is an $H$-separable extension of $B^{G}$.

(3) By (1) and (2), $B$ is a Galois $H$-separable extension of $B^{G}$.

(4) $\Delta=V_{B}\left(B^{G}\right)=Q[i]$ is not a Galois extension of $\Delta^{G}$ with Galois group $\left.G\right|_{\Delta} \cong G$, and so $B$ is not a central commutator Galois extension of $B^{G}$.

ACKNOWLEDGEMENT. This paper was written under the support of a Caterpillar Fellowship at Bradley University. We would like to thank Caterpillar Inc. for the support.

\section{REFERENCES}

[1] R. Alfaro and G. Szeto, On Galois extensions of an Azumaya algebra, Comm. Algebra 25 (1997), no. 6, 1873-1882. MR 98h:13007. Zbl 890.16017.

[2] F. R. DeMeyer, Some notes on the general Galois theory of rings, Osaka J. Math. 2 (1965), 117-127. MR 32\#128. Zbl 143.05602.

[3] _ Galois theory in separable algebras over commutative rings, Illinois J. Math. 10 (1966), 287-295. MR 33\#149. Zbl 216.34001.

[4] F. R. DeMeyer and E. Ingraham, Separable Algebras over Commutative Rings, vol. 181, Springer-Verlag, Berlin, New York, 1971, Lecture Notes in Mathematics. MR 43\#6199. Zbl 215.36602.

[5] M. Harada, Supplementary results on Galois extension, Osaka J. Math. 2 (1965), 343-350. MR 33\#151. Zbl 178.36903.

[6] T. Kanzaki, On Galois algebra over a commutative ring, Osaka J. Math. 2 (1965), 309-317. MR 33\#150. Zbl 163.28802.

[7] K. Sugano, On centralizers in separable extensions, Osaka J. Math. 7 (1970), 29-40. MR 42\#6048. Zbl 206.32502.

[8] _ On a special type of Galois extensions, Hokkaido Math. J. 9 (1980), no. 2, 123-128. MR 82c:16036. Zbl 467.16005.

[9] G. Szeto and L. Ma, On center-Galois extensions over a ring, Glas. Mat. Ser. III 24(44) (1989), no. 1, 11-16. MR 90j:16081. Zbl 686.16029.

[10] G. Szeto and L. Xue, On three types of Galois extensions of rings, Southeast Asian Bull. Math. 23 (1999), no. 4, 731-736. Zbl 991.19722.

[11] On Characterizations of a center Galois extension, Internat. J. Math. Math. Sci. 23 (2000), no. 11, 753-758.

George Szeto: Mathematics Department, Bradley University, Peoria, Illinois 61625, USA

E-mail address: szeto@brad1ey.brad1ey.edu

Lianyong Xue: Mathematics Department, Bradley University, Peoria, Illinois 61625, USA

E-mail address: 1xue@brad7ey.brad7ey.edu 


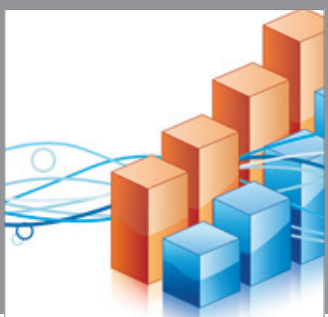

Advances in

Operations Research

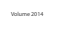

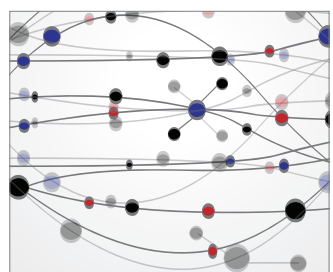

\section{The Scientific} World Journal
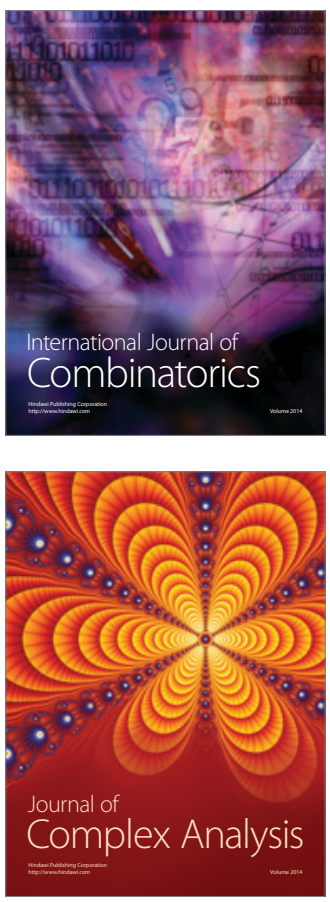

International Journal of

Mathematics and

Mathematical

Sciences
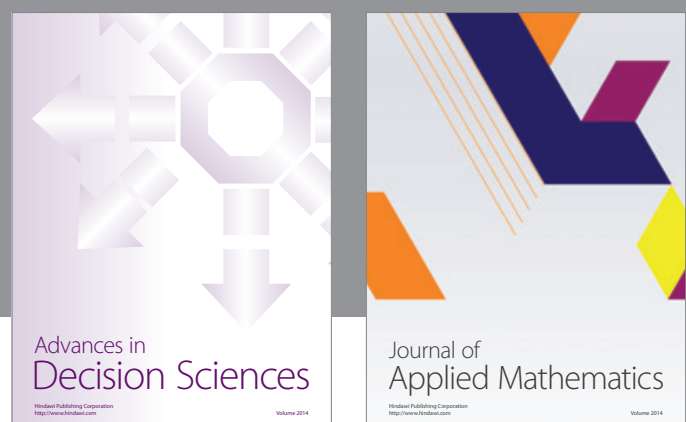

Journal of

Applied Mathematics
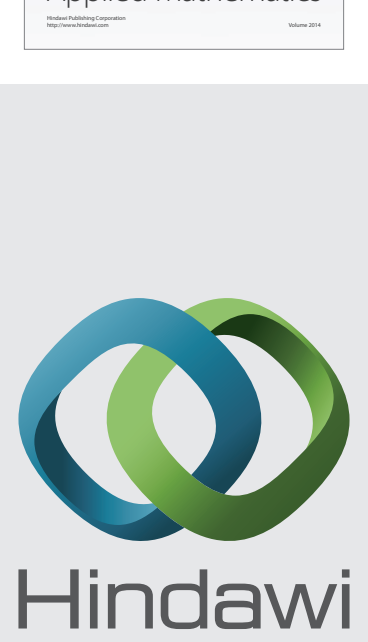

Submit your manuscripts at http://www.hindawi.com
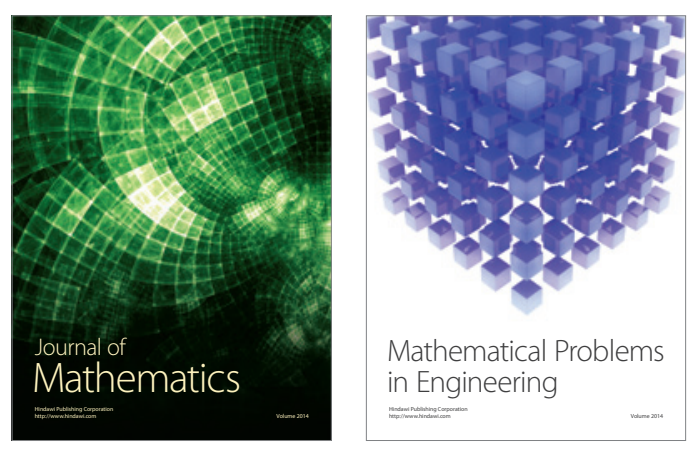

Mathematical Problems in Engineering
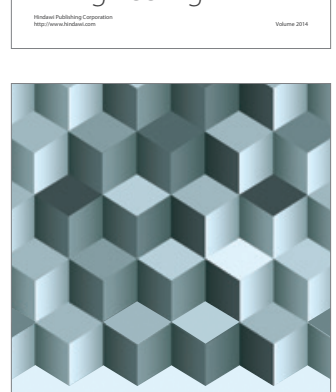

Journal of

Function Spaces
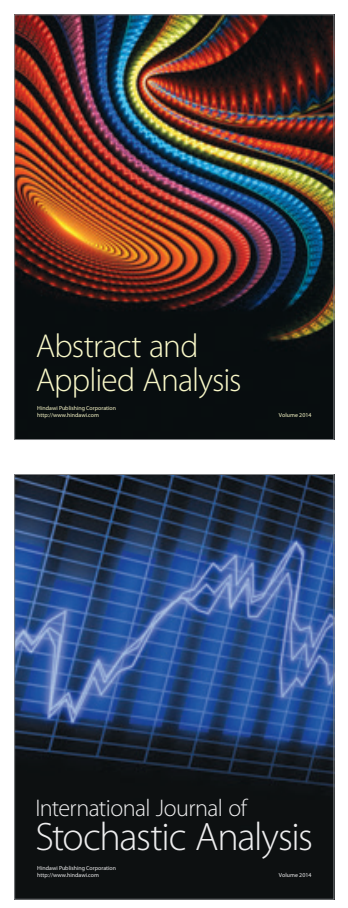

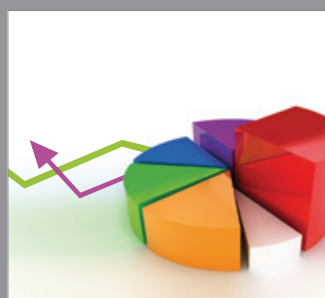

ournal of

Probability and Statistics

Promensencen
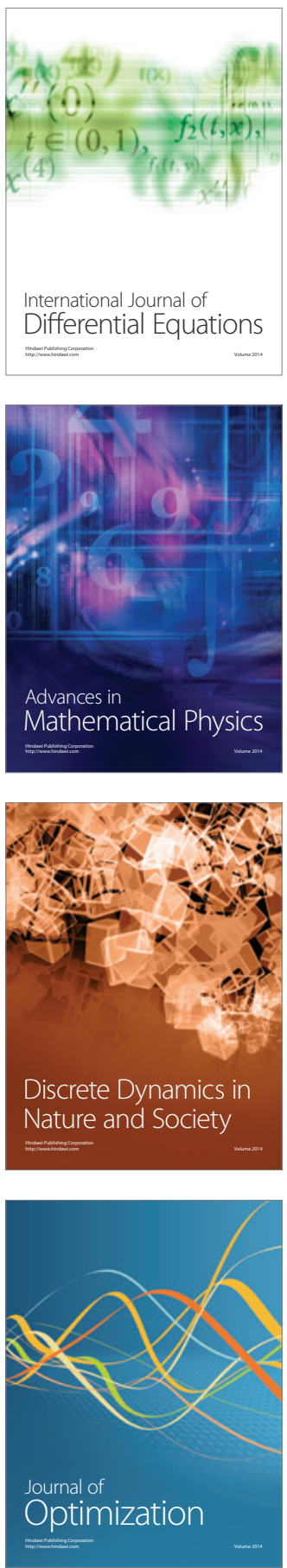